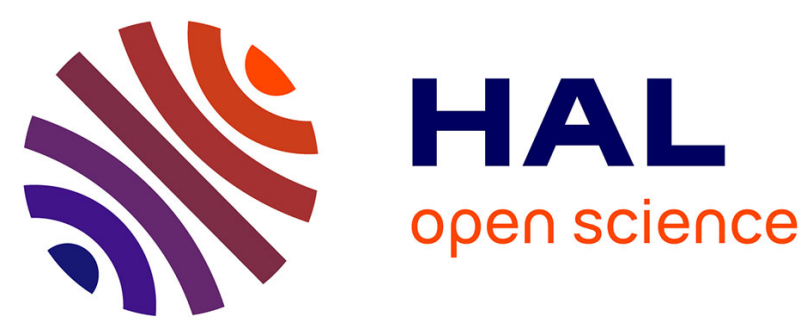

\title{
Benefits of immune protection versus immunopathology costs: a synthesis from cytokine KO models.
} Gabriele Sorci, Cédric Lippens, Clothilde Léchenault, Bruno Faivre

\section{To cite this version:}

Gabriele Sorci, Cédric Lippens, Clothilde Léchenault, Bruno Faivre. Benefits of immune protection versus immunopathology costs: a synthesis from cytokine KO models.. Infection, Genetics and Evolution, 2017, 54, pp.491-495. 10.1016/j.meegid.2017.08.014 . hal-01577453

\section{HAL Id: hal-01577453 \\ https://hal.science/hal-01577453}

Submitted on 21 Feb 2022

HAL is a multi-disciplinary open access archive for the deposit and dissemination of scientific research documents, whether they are published or not. The documents may come from teaching and research institutions in France or abroad, or from public or private research centers.
L'archive ouverte pluridisciplinaire HAL, est destinée au dépôt et à la diffusion de documents scientifiques de niveau recherche, publiés ou non, émanant des établissements d'enseignement et de recherche français ou étrangers, des laboratoires publics ou privés. 
Benefits of immune protection versus immunopathology costs:

3 a synthesis from cytokine $K O$ models

4

5 Gabriele Sorci*, Cédric Lippens, Clothilde Léchenault, Bruno Faivre

6

7 Biogéosciences, CNRS UMR 6282, Université de Bourgogne Franche-Comté, 6 boulevard Gabriel, 821000 Dijon, France

9

10

*Corresponding author: gabriele.sorci@u-bourgogne.fr

11 


\section{Abstract}

The inflammatory response can produce damage to host tissues and in several infectious diseases the most severe symptoms are due to immunopathology rather than a direct effect of pathogen multiplication. One hypothesis for the persistence of inflammatory damage posits that the benefits of protection towards infection outweigh the costs. We used data on knocked-out $(K O)$ cytokine models [and the corresponding wild-type (WT) controls] to test this hypothesis. We computed differences in pathogen load and host survival between $W T$ and $K O$ and divided them by the $W T$ values. Using this ratio provides an internal control for variation in pathogen species, host strain, pathogen dose, and inoculation route. We predicted that i) if mortality is essentially due to immunopathology, there should be a loose association between pathogen load and host survival; ii) if mortality is essentially due to pathogen proliferation, we expect a tight association between pathogen load and host survival. The results provide strong support to this latter hypothesis. In $85 \%$ of $W T-K O$ comparisons $(\mathrm{n}=126)$, an increase in pathogen load was associated with an increase in host mortality, and a decrease in pathogen load was associated with a decrease in host mortality. Overall, these findings are in agreement with the idea that immunopathology persists because immune protection confers immediate benefits in terms of infection clearance.

Key-words: cytokine, immunopathology, inflammation, pathogen load, host survival, virulence 30 


\section{Introduction}

By definition pathogens exploit resources provided by an unrelated organism, called host (Combes, 2001). Pathogen exploitation diverts resources from host functions and as such is considered costly for the host. Indeed, infection is usually associated with impaired host fitness, either in terms of increased mortality or decreased reproductive output (Schmid-Hempel, 2008). Such infectionassociated costs define virulence (Read, 1994). The evolution of virulence has been a puzzling question for evolutionary biologists for decades. Indeed, why should a pathogen that relies on host survival for its own survival kill its host? A solution to this paradox was provided a few decades ago when it was suggested that pathogen fitness does not depend on its survival but on its transmission to other susceptible hosts (Anderson and May, 1982; Ewald, 1983). The model, coined the trade-off model of virulence, assumes that pathogen transmission increases with pathogen multiplication within the host, and that host mortality increases with multiplication (Alizon et al., 2009). While pathogen multiplication (host exploitation) improves transmission rate, it can also induce host death. Pathogen fitness is therefore maximized at intermediate levels of multiplication (exploitation) (see for instance de Roode et al., 2008; Alizon and Michalakis, 2015). This model establishes a direct link between cost of infection and pathogen multiplication. However, the severity of disease is often unrelated to pathogen load (see Graham et al., 2005 for a review). In this case, the severity of disease symptoms is rather associated with an overreacting immune response. Immune costs, incurred following an infection, are usually referred to as immunopathology (Graham et al., 2005).

While theoretical work has shown that taking into account immune costs does not invalidate the trade-off model of the evolution of virulence (Day et al., 2007; Best et al., 2012), a pending question is why immunopathology persists despite the associated fitness costs for the host (Graham et al., 2005 ). Immunopathology might be an unavoidable consequence of immune activation and even though it potentially causes damage to the host, a lack of immune activation upon infection would produce even more dramatic fitness consequences for the host due to pathogen proliferation (Okin and Medzhitov 2012). In addition to this, while failure to keep pathogen proliferation under 
control incurs immediate costs (including mortality), several inflammatory diseases (but not all) have late-life onset (occur at relatively old ages), and early benefits are expected to outweigh late costs (Okin and Medzhitov 2012). While sound, this hypothesis has proved difficult to test over a large spectrum of host-pathogen systems. Here, we took advantage of published information on cytokineknocked out mouse models to test the idea that benefits of immune protection outweigh the immunopathology costs.

Cytokines are signaling molecules produced by different immune cells playing a central role in the immune response (Opal and DePalo, 2000; Scapligliati et al., 2006; Banchereau et al., 2012). Cytokines exhibit interesting properties such as synergy, redundancy, or inhibitory effects (Paludan, 1998; Elenkov et al. 2005). Based on the immune cells that produce them and on their action on the immune response, cytokines have been grouped and classified as having pro- or anti-inflammatory properties or skewing the immune response towards a Th1, Th2, or Th17 type. It is worth noting however, that not all cytokines clearly fall into one of these categories, and their anti- or proinflammatory role can also be context-dependent (Cavaillon, 2001).

As signaling molecules, cytokines play a central role in the process of resistance to infectious diseases, and they have received a lot of attention to identify how they promote clearance of infection (Guidotti and Chisari, 2000; Cooper et al., 2011; Beltra and Decaluwe, 2016). One useful tool to study the effect of cytokines on pathogen resistance has been to produce mouse strains where the gene that codes for a given cytokine has been inactivated or disrupted. Traits of interest of these knock out $(K O)$ mouse models are then compared to those of the corresponding wild type (WT) strains, in response to an infectious threat. This offers the opportunity to explore whether an intrinsically up or down-regulated immune response affects both pathogen proliferation and host fitness (i.e., survival).

We compiled a database comprising $19 K O$ cytokines (or cytokine receptors) from 86 published articles. This gives $126 W T-K O$ comparisons for differences in host survival and pathogen load, involving $21 \%$ of viruses, $52 \%$ bacteria, $6 \%$ fungi and $22 \%$ protozoa. Based on the observation 
that disease severity may not be correlated with pathogen load (Gazzinelli et al., 1996; Kullberg et al., 1998; Long et al., 2006; Long et al., 2008a,b; Long and Graham, 2011), we predicted that if cost of infection is due to immunopathology there should be no clear association between differences in host survival and differences in pathogen load. On the contrary, if cost of infection is essentially due to pathogen proliferation, we should expect a strong association between differences in host survival and differences in pathogen load.

\section{Material and methods}

\subsection{Data acquisition}

We searched the literature indexed in the Web of Science, using a list of key words (including 'KO', 'cytokine', 'infection', 'survival', 'mortality'). For each article, we checked that there was a cytokine $K O$ model (either the protein or the receptor), an infectious model (a viral, bacterial, fungal, or protozoan infection), a measure of pathogen load in both $K O$ and $W T$ hosts, the assessment of survival in $K O$ and $W T$ hosts. Some articles also reported the production of cytokines in $K O$ and $W T$ hosts in response to the infection (evidently not the cytokine that was knocked out).

Data on pathogen load, host survival and when available cytokine production, were extracted from published figures using the freely available software ImageJ and webplotdigitizer (http://arohatgi.info/WebPlotDigitizer/app/). For survival data, we computed the areas below the survival curves for $W T$ and $K O$ individuals. For pathogen load, when means were reported at different time points, these values were summed up to have a cumulative load. Similarly, when pathogen load was reported for several organs, we summed up the means to obtain an overall value per group (WT and $K O$ ). Table 1 reports the list of cytokines, their role (pro- or anti-inflammatory), and the number of articles used to extract the data. The full dataset is available as an online supplementary material. 


\subsection{Statistical analyses}

For each trait (host survival, pathogen load and cytokine production), we computed the difference between $W T$ and $K O$ and divided it by the $W T$ value $\left(\frac{W T-K O}{W T}\right)$. Even though computing this ratio controls for the differences in scale between measurements (which is a concern for measurements of pathogen load), we decided to avoid using linear models to analyze the data. Instead, we preferred using a semi-quantitative approach. These ratios have signs indicating whether $W T$ had more pathogens than $K O$ or suffered more mortality than $K O$. Signs are obviously totally independent from the measurement scale used in the original data. The ratios for host survival and pathogen load were plotted one against the other. This gives a four quadrants plot. Each of these quadrants refers to a particular case. Moving in a clockwise direction, quadrant 1 refers to the case where $W T$ have better survival and lower pathogen load than $K O$, quadrant 2 refers to the case where $W T$ have better survival and higher pathogen load than $K O$, quadrant 3 refers to the case where $W T$ have lower survival and higher pathogen load than $K O$, and quadrant 4 refers to the case where $W T$ have lower survival and lower pathogen load than $K O$. Figure 1 reports a schematic illustration of the four quadrants and their meaning. We then tested the distribution of these ratios across the different quadrants using a $\chi^{2}$ test. If host survival and pathogen load are uncoupled, we expect to observe an homogenous distribution of points across the different quadrants $(25 \%$ of points in each quadrant); if pathogen load and host survival are negatively associated we expect to find that the majority of points lies in the diagonal across quadrants 1 and 3.

\section{Results}

In the vast majority of cases, suppressing pro-inflammatory cytokines increased pathogen load, since $83 \%$ of the ratios were negative $\left(\chi^{2}{ }_{1}=48.00, p<0.0001, n=108\right.$, fig. $\left.2 A\right)$. Sixty one percent of the pathogen load ratios were positive when anti-inflammatory cytokines were suppressed. However, this was not significantly different from the random distribution (50\% positive, $50 \%$ negative) $\left(\chi^{2}{ }_{1}=0.89, p=0.346, n=18\right.$, fig. $\left.2 B\right)$. The difference in distribution of ratio signs between 
pro- and anti-inflammatory cytokines was, however, highly significant $\left(\chi^{2}{ }_{1}=17.20, p<0.0001, n=\right.$ 126).

We found that the distribution of points across the four quadrants was not random $\left(\chi^{2}{ }_{3}=\right.$ 137.05, $\mathrm{p}<0.0001, \mathrm{n}=126$, fig. $3 \mathrm{~A})$. In agreement with the hypothesis that host survival is tightly negatively associated with pathogen load, we found that $85 \%$ of the data points laid in the quadrants along the diagonal (quadrants 1 and 3 ). This pattern was even stronger when computing mean values per cytokine, since $95 \%$ of mean values were in quadrants 1 and $3\left(\chi^{2}{ }_{1}=15.21, p<0.0001, n=19\right.$, fig. 3B).

The distribution of ratio values across the four quadrants differed between pro- and antiinflammatory cytokines $\left(\chi^{2}{ }_{3}=18.41, p=0.0004, n=126\right.$, fig. $\left.3 A\right)$. While $75 \%$ of points involving proinflammatory cytokines laid in quadrant 1 vs. 39\% for anti-inflammatory cytokines, only $10 \%$ of proinflammatory cytokines were in quadrant 3 vs. $44 \%$ for anti-inflammatory cytokines (fig. 3A). This reflects the general trend that silencing pro-inflammatory cytokines enhanced pathogen load and mortality in $K O$ individuals.

The distribution of ratio values across the four quadrants was remarkably consistent when comparing cytokine vs. receptor $K O$ models, or over the three major pathogen taxonomic groups (viruses, bacteria and protozoa) (see online supplementary material, figures S1 and S2).

We also explored the possible compensatory effects induced by knocking out proinflammatory cytokines. Here, two possible predictions can be made. First, failure in producing a given pro-inflammatory cytokine might induce the over-expression of other cytokines with redundant functions, therefore producing a compensatory effect. Alternatively, knocking out proinflammatory cytokine genes might have pleiotropic effects on other signaling molecules. We did not find support for any of these hypotheses since in $55 \%$ of the cases silencing a pro-inflammatory cytokine induced the up-regulation of other pro-inflammatory cytokines and in $45 \%$ it produced a down-regulation $\left(\chi^{2}{ }_{1}=1.17, \mathrm{p}=0.278, \mathrm{n}=103\right)$. Silencing an anti-inflammatory cytokine almost 
always produced an up-regulation of pro-inflammatory cytokines (in $92 \%$ of cases, $\chi^{2}{ }_{1}=9.31, p=$ $0.0023, n=13)$.

\section{Discussion}

Using a compiled database from published literature we show that, by far, changes in pathogen load are the most important determinant of cost of infection, assessed as host mortality, in cytokine $K O$ mouse models. This finding shows that immediate benefits of infection clearance outweigh the, possibly delayed, immunopathology costs. This strongly suggests that immunopathology costs persist in face of natural selection because immune protection confers immediate benefits in terms of survival.

Traditionally, evolutionary biologists saw virulence (host mortality or impaired health) as the unavoidable consequence of within-host pathogen multiplication, and within-host multiplication as a trait that increases pathogen transmission rate (Alizon and Michalakis, 2015). This view therefore establishes a direct link between pathogen load and host mortality risk (or health impairment). On the contrary, biomedical sciences have, since long, stressed the idea that mounting an immune response against an infectious organism can produce devastating collateral effects, possibly more severe than the direct damage due to resource exploitation by the pathogen (Lambrecht and Hammad 2012; Sharma and Thomas, 2014; Newton et al., 2016; Shin et al., 2016). Text book examples of such infection-induced immunopathology costs involve cerebral malaria or respiratory distress during influenza (Peiris et al., 2010; Schmidt et al., 2011; Damjanovic et al., 2012). Since the seminal paper published by Graham and coworkers in 2005 (Graham et al., 2005), evolutionary biologists have started to take into consideration how immunopathology might affect the evolution of parasite virulence and host defenses (Sadd and Siva-Jothy 2006; Belloni et al., 2010; Cressler et al., 2015). While theoretical work has overall shown that the general conclusions of the trade-off model of parasite virulence hold even when host death is due to immune-mediated diseases (Day et al., 2007; Best et al., 2012), we still have little insights on the evolutionary forces that maintain 
immunopathology. Pioneering work conducted on rodent malaria has provided a detailed assessment of the relative role of immunopathology and pathogen load as drivers of disease severity. Using a combination of experimental infections with different clones of the protozoan Plasmodium chabaudii and the blockade of pro- (TNF- $\alpha$ ) or anti-inflammatory (IL-10) effectors in infected mice, Long and coworkers were able to drive virulence (infection-induced mortality) up and down (depending on the immune treatment used), independently of pathogen load (Long et al., 2008a,b). These examples show that immune activation contributes to the cost of infection and that this cost can be decoupled from pathogen load.

Although the idea that immunopathology is the unavoidable consequence of immune protection makes perfectly sense (Okin and Medzhitov, 2012), to our knowledge no quantitative study, on a broad range of infectious organisms, has been produced to support or invalidate this idea. Here, we show that inactivating pro-inflammatory cytokine genes increases parasite load and host mortality over a large spectrum of disease models. This produces a tight association between pathogen load and host mortality, in agreement with the hypothesis that uncontrolled pathogen proliferation induces immediate and potentially devastating costs for the host. Inflammatory diseases are often associated with up-regulation of pro-inflammatory cytokines and failure to shutdown the production of inflammatory effectors (Wallace et al., 2014). Therefore, immunopathology might be removed by down-regulating pro-inflammatory cytokines (Gottschalk et al., 2016; Liu et al., 2016); however, it is likely that the associated overwhelming pathogen proliferation weakens the adaptive value of reducing the expression of pro-inflammatory effectors.

Although the strength of our study is that it involves different immune effectors and pathogens, this also raises a series of challenges. Obviously the first challenge is that it is difficult to compare studies that used different pathogenic organisms that have been inoculated to different mouse strains, at different doses, using different inoculation modes, monitored over different periods of time, etc. We got rid of all these confounding effects by using $W T$ individuals as the internal control. Even if studies differ in many aspects, within studies, $K O$ and $W T$ mice were always 
exposed to the same inoculum size, monitored over the same period of time and so on. Therefore, computing the differences between $W T$ and $K O$ and dividing them by the $W T$ values, gives an objective estimate of the relative effect of knocking out a given cytokine on host survival and pathogen load. To be even more conservative, we decided to refrain from using linear models to explore the relationship between pathogen load and host mortality, because of the possible effect of different scales used to assess pathogen load across infectious organisms. The method that we adopted, based on the distribution of the signed ratios, does not allow us to quantitatively estimate the fraction of variance in host survival accounted by pathogen load; nevertheless, it unambiguously shows that variation in pathogen load (up or down, depending on the type of cytokine knocked out) is associated with variation in host mortality. It should also be made clear that the computation of these ratios does not take into account whether the original study reported a statistically significant difference or not. Actually, in several studies pathogen load or host survival were indeed not significantly different between $W T$ and $K O$. However, if the signed ratios were mostly due to random variation around a zero mean, we should have found a homogenous distribution of the data points across the four quadrants. this issue, we investigated whether there was a temporal trend in the data by looking at the variation in the ratios among years and we did not find any consistent trend (see online supplementary material, figures S3). In addition to this, the reported association is so strong that the publication bias should be very substantial to mask the observed pattern, which seems rather unlikely. pathogen load, we do not discard the role of immunopathology as an important component of infection cost. In particular, to stick with the definition of virulence (impairment of host fitness), we focused on studies dealing with pathogens that do induce host mortality (almost no studies in the context of $K O$ infected models have investigated reproductive output) and therefore are amenable for the study of the immediate cost of infection. Several pathogen species are not immediately lethal 
and instead have more diffuse and long term costs. Further work should include such infectious models to have an even more comprehensive view on the benefits of immune protection vs. the costs of immunopathology. We are grateful to Aurélie Tasiemski for helpful discussions.

Cytokine signaling is characterized by properties such as redundancy, synergy, pleiotropy, inhibition (Elenkov et al., 2005; Cavaillon, 2001). Silencing genes coding for a given pro-inflammatory cytokine might therefore induce a compensatory effect involving other cytokines with redundant properties. The finding that mice with $K O$ pro-inflammatory cytokines overall suffered from increased pathogen load and mortality suggests that, if any, the compensatory effect is far from being perfect. To go further in this direction, we investigated whether $K O$ individuals had an up-regulated expression of some of the main pro-inflammatory cytokines (TNF- $\alpha$, IFN- $\gamma$, IL-1 $\beta$, IL-17). We found no consistent pattern since in half of the cases $K O$ individuals had down-regulated expression of proinflammatory cytokines, and the other half an up-regulated expression. On the contrary, knocking out anti-inflammatory cytokines consistently produced an up-regulation of pro-inflammatory cytokines. This latter finding is in agreement with the prominent role that anti-inflammatory cytokines play in the process of resolution of the inflammatory response.

To conclude, we provide evidence, based on a diverse set of infectious organisms that even though suppressing pro-inflammatory signaling reduces infection-associated immunopathology, pathogen proliferation incurs an immediate major fitness cost in terms of mortality risk. The balance between immediate cost of pathogen proliferation and delayed cost of immunopathology therefore provides an evolutionary explanation for the persistence of immunopathology (Okin and Medzhitov, 2012). 


\section{References}

Alizon, S., Hurford, A., Mideo, N., van Baalen, M., 2009. Virulence evolution and the trade-off hypothesis: history, current state of affairs and the future. J. evol. Biol. 22, 245-259.

Alizon, S., Michalakis, Y., 2015. Adaptive virulence evolution: the good old fitness-based approach. Trends Ecol. Evol. 30, 248-254.

Anderson, R.M., May, R.M., 1982. Coevolution of hosts and parasites. Parasitology 85, 411-426.

Banchereau, J., Pascual, V., O'Garra, A., 2012. From IL-2 to IL-37: the expanding spectrum of antiinflammatory cytokines. Nature Immunol. 13, 925-931.

Belloni, V., Faivre, B., Guerreiro, R., Arnoux, E., Bellenger, J., Sorci, G., 2010. Suppressing an antiinflammatory cytokine reveals a strong age-dependent survival cost in mice. PLoS ONE 5, e12940.

Beltra, J.C., Decaluwe, H., 2016. Cytokines and persistent viral infections. Cytokine 82, 4-15.

Best, A., Long, G., White, A., Boots, M., 2012. The implications of immunopathology for parasite evolution. Proc. R. Soc. B 279, 3234-3240.

Cavaillon, JM., 2001. Pro- versus anti-inflammatory cytokines: myth or reality. Cell. Mol. Biol. 47, 695702.

Combes, C., 2001. Parasitism: The ecology and evolution of intimateinteractions. University of Chicago Press.

Cooper, A.M., Mayer-Barber, K.D., Sher, A., 2011. Role of innate cytokines in mycobacterial infection. Mucosal Immunol. 4, 252-260.

Cressler, C.E., Graham, A.L., Day, T., 2015. Evolution of hosts paying manifold costs of defence. Proc. R. Soc. B 282, 20150065.

Damjanovic, D., Small, C.L., Jeyananthan, M., McCormick, S., Xing, Z., 2012. Immunopathology in influenza virus infection: Uncoupling the friend from foe. Clin. Immunol. 144, 57-69.

Day, T., Graham, A.L., Read, A.F., 2007. Evolution of parasite virulence when host responses cause disease. Proc. R. Soc. B 274, 2685-2692. 
de Roode, J.C., Yates, A.J., Altizer, S., 2008. Virulence transmission trade-offs and population divergence in virulence in a naturally occurring butterfly parasite. Proc. Natl. Acad. Sci. USA $105,7489-7494$.

Elenkov, I.J., lezzoni, D.G., Daly, A., Harris, A.G., Chrousos, G.P., 2005. Cytokine dysregulation, inflammation and well-being. Neuroimmunomodulation 12, 255-269.

Ewald, P.W., 1983. Host-parasite relations, vectors, and the evolution of disease severity. Ann. Rev. Ecol. Syst. 14, 465-485.

Gazzinelli, R.T., Wysocka, M., Hieny, S., Scharton-Kersten, T., Cheever, A., Kuhn, R., Muller, W., Trinchieri, G., Sher, A., 1996. In the absence of endogenous IL-10, mice acutely infected with Toxoplasma gondii succumb to a lethal immune response dependent on CD4+ T cells and accompanied by overproduction of IL-12, IFN-gamma and TNF-alpha. J. Immunol. 157, 798805.

Gottschalk, T.A., Tsantikos, E., Hibbs, M.L., 2016. Pathogenic inflammation and its therapeutic targeting in systemic lupus erythematosus. Front. Immunol. 6, 550.

Graham, A.L., Allen, J.E., Read, A.F., 2005. Evolutionary causes and consequences of immunopathology. Ann. Rev. Ecol. Syst. 36, 373-397.

Guidotti, L.G., Chisari, F.V., 2000. Cytokine-mediated control of viral infections. Virology 273, 221227.

Kullberg, M.C., Ward, J.M., Gorelick, P.L., Caspar, P., Hieny, S., Cheever, A., Jankovic, D., Sher, A., 1998. Helicobacter hepaticus triggers colitis in specific-pathogen-free interleukin-10 (IL-10)deficient mice through an IL-12- and gamma interferon-dependent mechanism. Infect. Immun. $66,5157-5166$.

Lambrecht, B.N., Hammad, H., 2012. Lung dendritic cells in respiratory viral infection and asthma: From protection to immunopathology. Ann. Rev. Immunol. 30, 243-270.

Liu, Q., Zhou, Y.H., Yang, Z.Q., 2016. The cytokine storm of severe influenza and development of immunomodulatory therapy. Cell. Mol. Immunol. 3, 3-10. 
Long, G.H., Chan, B.H.K., Allen, J.E., Read, A.F., Graham, A.L., 2006. Parasite genetic diversity does not influence TNF-mediated effects on the virulence of primary rodent malaria infections. Parasitology 133, 673-684.

Long, G.H., Chan, B.H.K., Allen, J.E., Read, A.F., Graham, A.L., 2008a. Experimental manipulation of immune-mediated disease and its fitness costs for rodent malaria parasites. BMC Evol. Biol. 8, 128.

Long, G.H., Chan, B.H.K., Allen, J.E., Read, A.F., Graham, A.L., 2008b. Blockade of TNF receptor 1 reduces disease severity but increases parasite transmission during Plasmodium chabaudi chabaudi infection. Int. J. Parasitol. 38, 1073-1081.

Long, G.H., Graham, A.L., 2011. Consequences of immunopathology for pathogen virulence evolution and public health: malaria as a case study. Evol. Appl. 4, 278-291.

Newton, A.H., Cardani, A., Braciale, T.J., 2016. The host immune response in respiratory virus infection: balancing virus clearance and immunopathology. Semin. Immunopathol. 38, 471482.

Okin, D., Medzhitov, R., 2012. Evolution of inflammatory diseases. Curr. Biol. 22, R733-R740.

Opal, S.M., DePalo, V.A., 2000. Anti-inflammatory cytokines. Chest 117, 1162-1172.

Paludan, S.R., 1998. Interleukin-4 and Interferon- $\gamma$ : the quintessence of a mutual antagonistic relationship. Scand. J. Immunol. 48, 459-468.

Peiris, J.S.M., Hui, K.P.Y., Yen, H.L., 2010. Host response to influenza virus: protection versus immunopathology. Curr. Opin. Immunol. 22, 475-481.

Read, A.F., 1994. The evolution of virulence. Trends Microbiol. 2, 73-76.

Sadd, B.M., Siva-Jothy, M.T., 2006. Self-harm caused by an insect's innate immunity. Proc. R. Soc. B $273,2571-2574$.

Scapigliati, G., Buonocore, F., Mazzini, M., 2006. Biological activity of cytokines: an evolutionary perspective. Curr. Pharm. Des. 12, 3071-3081. 
Schmid-Hempel, P., 2008. Evolutionary parasitology: The integrated study of infections, immunology, ecology, and genetics. Oxford University Press.

Schmidt, K.E., Schumak, B., Specht, S., Dubben, B., Limmer, A., Hoerauf, A., 2011. Induction of proinflammatory mediators in Plasmodium berghei infected BALB/c mice breaks blood-brainbarrier and leads to cerebral malaria in an IL-12 dependent manner. Microbes Infect. 13, 828836.

Sharma, S., Thomas, P.G., 2014. The two faces of heterologous immunity: protection or immunopathology. J. Leukoc. Biol. 95, 405-416.

Shin, E.C., Sung, P.S., Park, S.H., 2016. Immune responses and immunopathology in acute and chronic viral hepatitis. Nature Rev. Immunol. 16, 509-523. 
Table 1.

360 List of $K O$ cytokines considered in the study, their role (pro- or anti-inflammatory), and the number of

361 articles from which data were extracted.

362

\begin{tabular}{|l|c|c|}
\hline \multicolumn{1}{|c|}{ KO cytokine } & Cytokine type & $\begin{array}{c}\text { Number } \\
\text { of articles }\end{array}$ \\
\hline IL-1 & Pro-inflammatory & 3 \\
\hline IL-1 receptor & Pro-inflammatory & 8 \\
\hline IL-3 & Pro-inflammatory & 2 \\
\hline IL-4 & Anti-inflammatory & 5 \\
\hline IL-4 receptor & Anti-inflammatory & 1 \\
\hline IL-5 & Anti-inflammatory & 1 \\
\hline IL-6 & Pro-inflammatory & 7 \\
\hline IL-10 & Anti-inflammatory & 10 \\
\hline IL-12 & Pro-inflammatory & 6 \\
\hline IL-15 & Pro-inflammatory & 2 \\
\hline IL-17 & Pro-inflammatory & 1 \\
\hline IL-17 receptor & Pro-inflammatory & 5 \\
\hline IL-18 & Pro-inflammatory & 7 \\
\hline IL-18 receptor & Pro-inflammatory & 1 \\
\hline IL-21 & Pro-inflammatory & 2 \\
\hline IL-21 receptor & Pro-inflammatory & 1 \\
\hline IL-22 & Pro-inflammatory & 2 \\
\hline IL-23 & Pro-inflammatory & 5 \\
\hline IL-27 receptor & Pro-inflammatory & 2 \\
\hline IL-33 receptor & Pro-inflammatory & 1 \\
\hline IL-36 receptor & Pro-inflammatory & 1 \\
\hline GM-CSF & Pro-inflammatory & 2 \\
\hline IFN & Pro-inflammatory & 17 \\
\hline IFN receptor & Pro-inflammatory & 7 \\
\hline TNF & Pro-inflammatory & 7 \\
\hline TNF receptor & \\
\hline
\end{tabular}


Figure 1. Schematic illustration of the method used to assess the association between differences in pathogen load and differences in host survival in $W T$ and $K O$ mice. Plotting the differences between $W T$ and $K O$ (divided by the $W T$ values) defines a four quadrant space, with each quadrant indicating a particular combination of differences between the two variables (pathogen load and host survival). In the absence of association between pathogen load and host survival, the distribution of data points

372 should be equal across the four quadrants. This random distribution represents the null hypothesis

$373\left(H_{0}\right)$ against which the observed distribution of data points is tested.

Figure 2. Difference in pathogen load between $W T$ and $K O$ mice divided by $W T$ pathogen load. A) genes coding for pro-inflammatory cytokines were inactivated; B) genes coding for anti-inflammatory 377 cytokines were inactivated.

378

379 Figure 3. Association between difference in host survival and pathogen load in WT and KO divided by 380 WT values. A) Empty and full dots refer to pro- and anti-inflammatory KO cytokines, respectively. B) 381 Dots refer to the mean values per $K O$ cytokine $( \pm S E)$. 


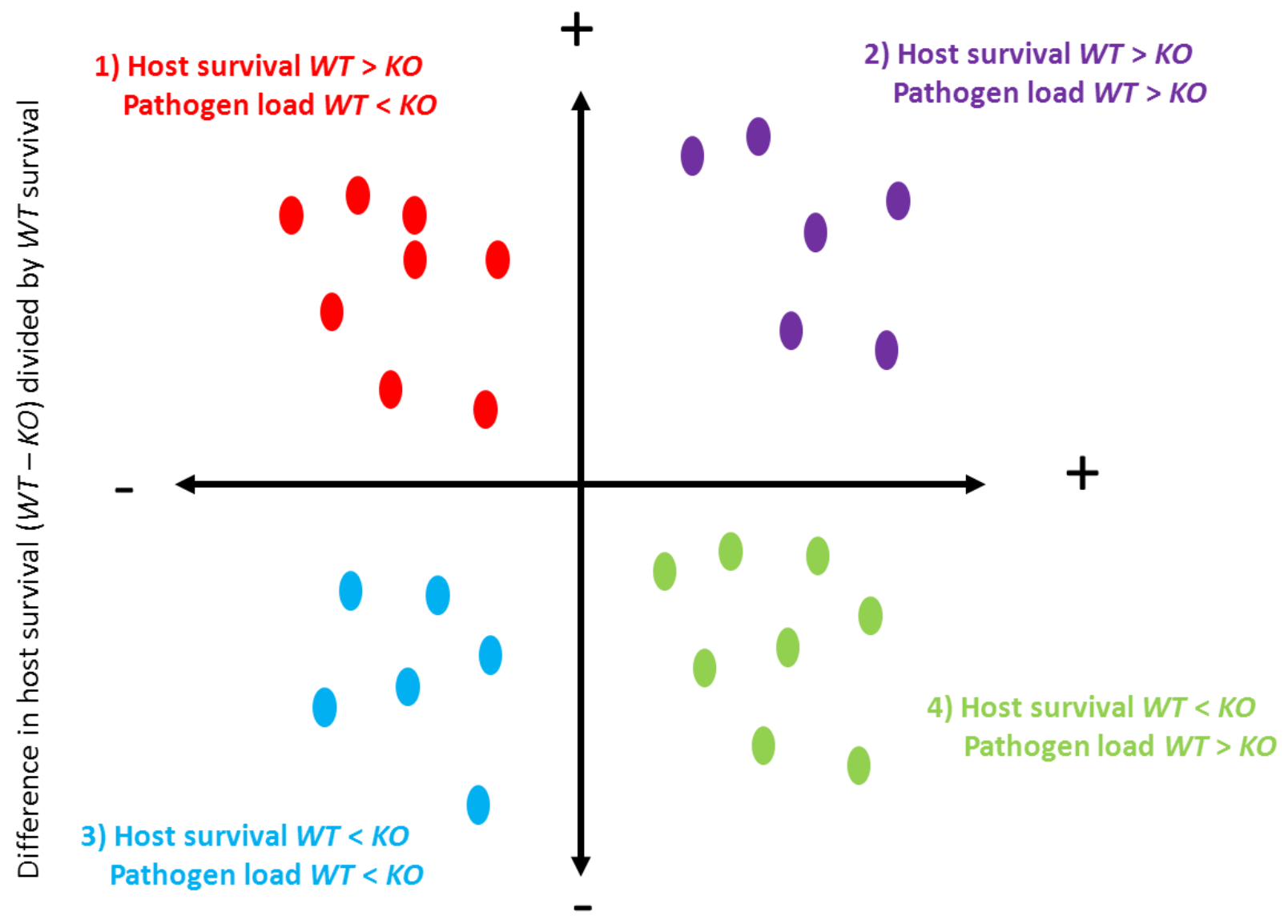

Difference in pathogen load $(W T-K O)$ divided by $W T$ pathogen load 


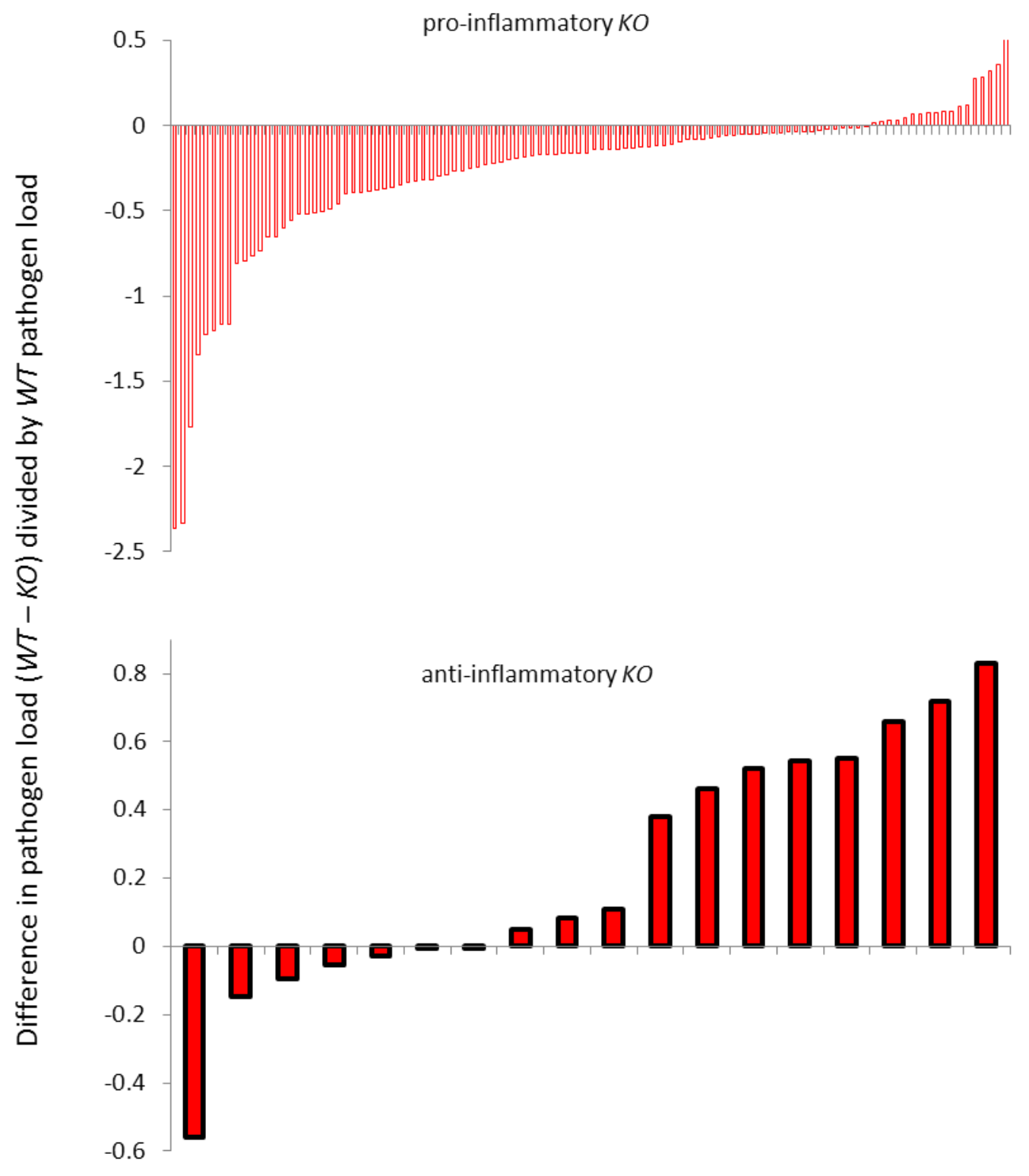

399

400

401

402

403

404

405

406 


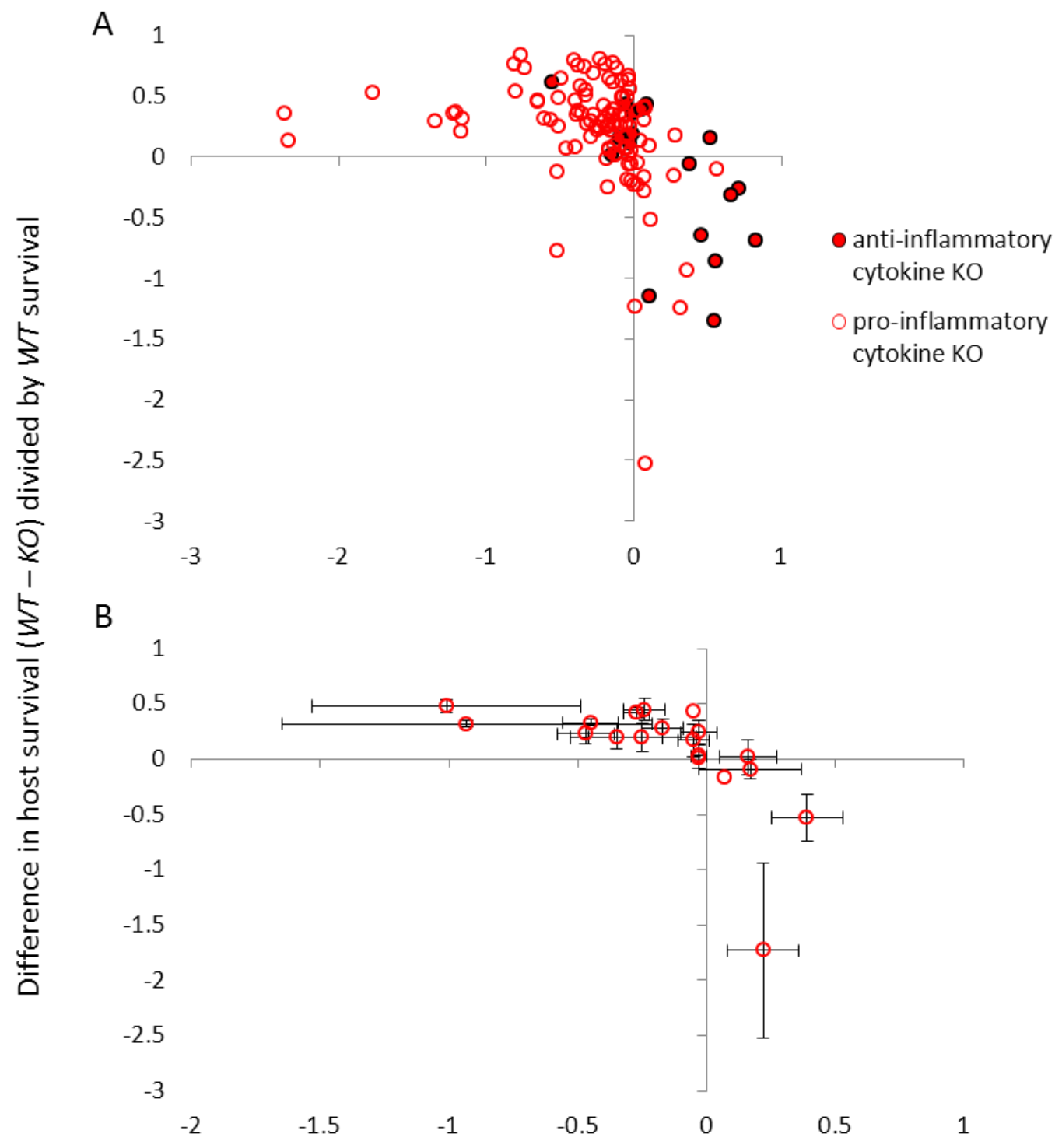

Difference in pathogen load $(W T-K O)$ divided by $W T$ pathogen load 